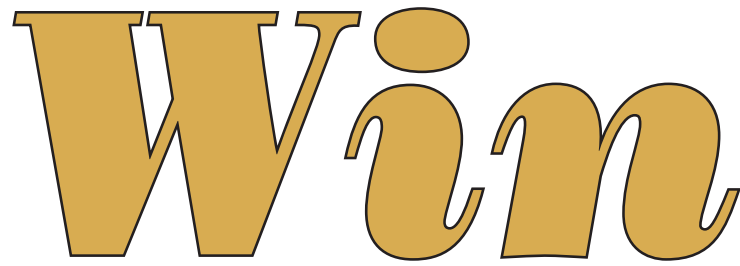

\title{
a magnum of Laurent-Perrier Champagne!
}

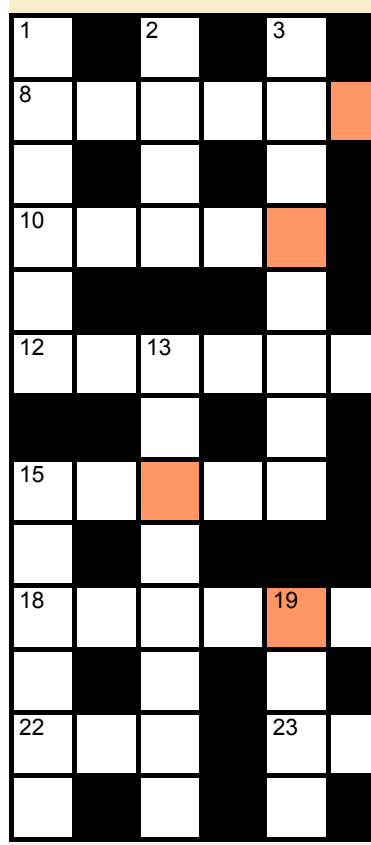

Across

8 Exploit, escapade (9)

9 Dull routine (3)

10 Wash out, swill (5)

11 In accordance with correct reasoning (7)

12 Welsh city (7)

14 Construct (5)

15 Provide food etc for a function (5)

16 Pick up (7)

18 The next one you'll come to (7)

20 Fox's burrow (5)

22/14dn. Atheist, agnostic (3-8)

23 University teacher (9)

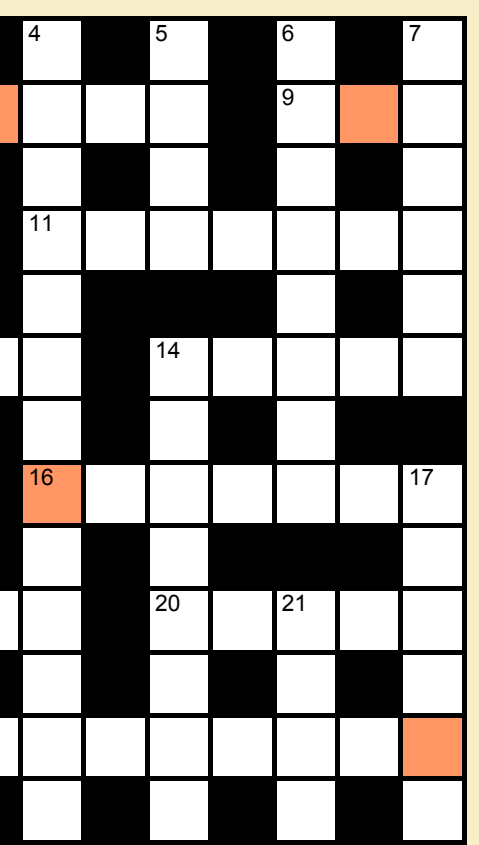

Down

1 Material, cloth (6)

2 Smooth, flat (4)

3 Below standard (8)

4 Certificate, etc of competence in a particular field or skill (13)

5 Hill, mountain or name of composer (4)

6 Dentist's business? (8)

7 Impassive, unemotional (6)

13 Does a course again? (8)

14 See $22 a c$.

15 Tooth: man's best friend? (6)

17 A casual top (1-5)

19 Catch sight of (4)

21 A sign of measles (4)
Rearrange the letters in the gold squares to find the secret word (7). Complete the answer form and send to the following address by 19 April 2013: Vital, BDJ Editorial, NPG, 4-6 Crinan Street, London, N1 9XW.

Racheal Lee, a dental nurse from Kingsteignton, won the winter Secretword (see below). The secret word was HYGIENE.

The answers and name of the prize winner will be announced on 8 June 2013 , when the summer issue of Vital is published.
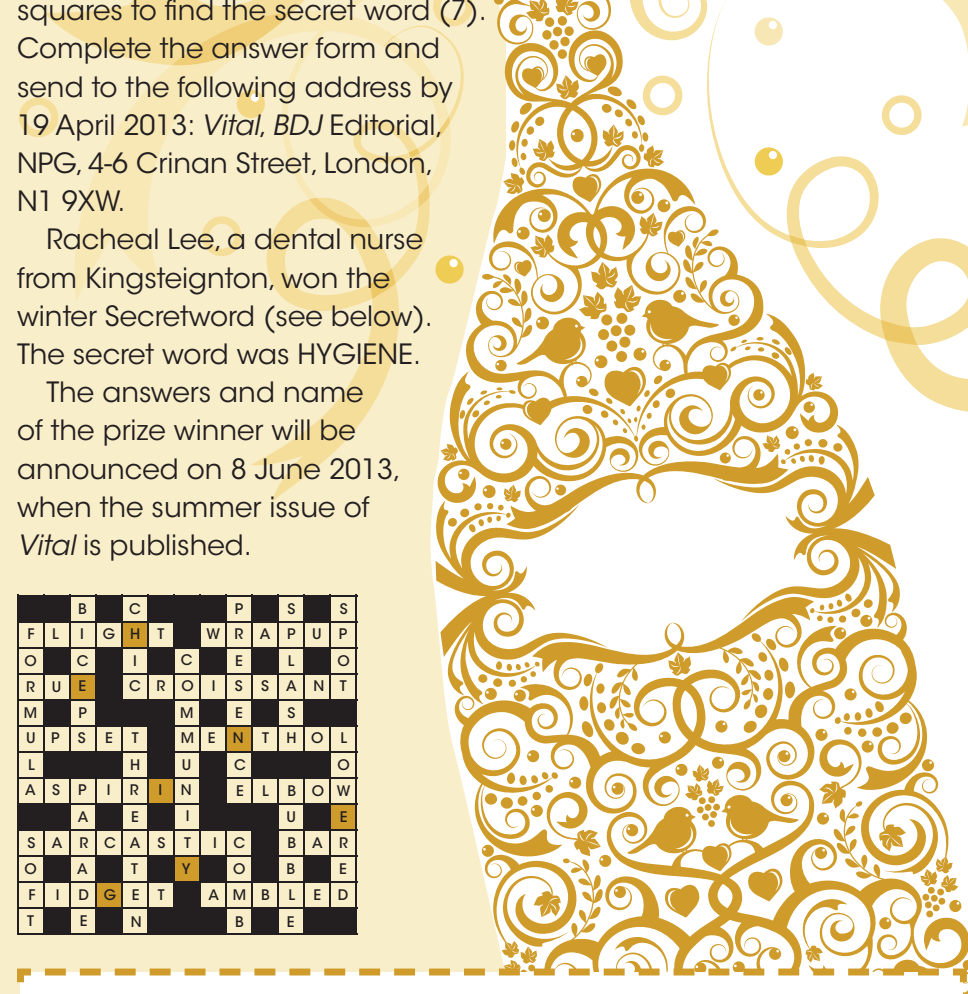
TITLE FIRST NAME

LAST NAME

ADDRESS I

POSTAL CODE 1 EMAIL

JOB TITLE

\section{SECRET WORD}

$\square$ If you prefer not to be contacted for purposes other than this competition, please tick this box. 